\title{
On a New Equation for Critical Current Density Directly in Terms of the BCS Interaction Parameter, Debye Temperature and the Fermi Energy of the Superconductor
}

\author{
G. P. Malik* \\ Theory Group, School of Environmental Sciences, Jawaharlal Nehru University, New Delhi, India. \\ Email: gulshanpmalik@yahoo.com,malik@mail.jnu.ac
}

Received February $7^{\text {th }}, 2013$; revised March $11^{\text {th }}, 2013$; accepted March $29^{\text {th }}, 2013$

Copyright (C) 2013 G. P. Malik. This is an open access article distributed under the Creative Commons Attribution License, which permits unrestricted use, distribution, and reproduction in any medium, provided the original work is properly cited.

\begin{abstract}
Recasting the BCS theory in the larger framework of the Bethe-Salpeter equation, a new equation is derived for the temperature-dependent critical current density $j_{c}(T)$ of an elemental superconductor (SC) directly in terms of the basic parameters of the theory, namely the dimensionless coupling constant $[\mathrm{N}(0) \mathrm{V}]$, the Debye temperature $\theta_{D}$ and, additionally, the Fermi energy $E_{F}$ - unlike earlier such equations based on diverse, indirect criteria. Our approach provides an $a b$ initio theoretical justification for one of the latter, text book equations invoked at $T=0$ which involves Fermi momentum; additionally, it relates $j_{c}$ with the relevant parameters of the problem at $T \neq 0$. Noting that the numerical value of $E_{F}$ of a high- $T_{c}$ SC is a necessary input for the construction of its Fermi surface-which sheds light on its gap-structure, we also briefly discuss extension of our approach for such SCs.
\end{abstract}

Keywords: Critical Current Density; BCS Parameters; Fermi Energy; Elemental/Non-Elemental Superconductors

\section{Introduction}

The critical current density $\left(j_{c}\right)$ of a superconductor (SC) is the maximum current density that it can carry beyond which it loses the characteristic of superconductivity. It is an important parameter because greater its value, greater is the practical use to which the SC can be put. The basic relation between $j_{c}$ and the critical velocity $\left(v_{c}\right)$ of Cooper pairs (CPs) at any temperature $T$ and an applied field $H$ is:

$$
j_{c}(T, H)=n_{s}(T, H) e^{*} v_{c}(T, H),\left(v_{c}=P_{c} / 2 m^{*}\right)
$$

where $n_{s}$ is the number of CPs, $e^{*}, P_{c}$ and $\left(2 m^{*}\right)$ are, respectively, the charge, the critical momentum, and the effective mass of a CP. We note that, since formation of CPs in the BCS theory is synonymous with the formation of their condensate [e.g., 1], $\mathrm{P}_{\mathrm{c}}$ in (1) may also be defined as the minimum momentum that causes dissociation of the condensate.

As alternatives to (1), several derived relations for $j_{c}$ can be found in the literature [2-5], some of which have been reproduced in Table 1. Salient features of these relations are: 1) They are obtained via indirect approaches based on diverse criteria such as the type of SC being dealt

*Present address: B-208 Sushant Lok I, Gurgaon 122002, Haryana, India. with (type I or II) and its geometry; 2) They lead to values of $j_{c} s$ that are generally much greater than the experimental values; and 3) Only one of them involves the Fermi energy $E_{F}$ (via Fermi momentum) of the $\mathrm{SC}$-this will be further discussed below.

$E_{F}$ of an $\mathrm{SC}$ is an important parameter too because, as has been remarked [6], "There is every evidence that the remarkable low value of $E_{F}(<100 \mathrm{meV})$ and the strong coupling of carriers with high-frequency phonons is the cause of high $T_{c}$ in all newly discovered superconductors." Furthermore, the input of the numerical value of $\mathrm{E}_{\mathrm{F}}$ is essential to construct the Fermi surface $E_{j}(k)$ of an SC via $E_{j}(k)=E_{F}$, from which it is seen that [7; p. 117] the whole process of determining theoretically the shape of the Fermi surface involves calculating $E_{j}(k)$ over the entire Brillouin zone and then constructing the particular constant energy surface that corresponds to $E_{F}$. However, this assumes that the actual numerical value of $E_{F}$ is available, which may well not be the case. The importance of the Fermi surface stems from the fact it sheds light on the gap-structure of the SC since it marks the boundary between the occupied and the unoccupied parts of the band $j$. This explains the considerable experimental effort that has been expended on constructing the 
Table 1. Some of the relations in the literature for calculating the critical current densities $\left(j_{c} s\right)$ of different types of superconductors obtained via diverse, indirect methods.

\begin{tabular}{|c|c|c|c|c|}
\hline S. No. & $\mathrm{SC}$ & $j_{c}$ & Remark & Ref. \\
\hline 1 & $\begin{array}{l}\text { Type I; wire of radius } a \text { in the } \\
\text { absence of external field }\end{array}$ & $\begin{array}{c}H_{c} a c / 2 \\
H_{c}: \text { critical magnetic field } \\
c: \text { velocity of light }\end{array}$ & $\begin{array}{l}j_{c} \text { is the current that generates } \\
\text { a field }=H_{c}\end{array}$ & \\
\hline 2 & Type I; thin film or wire & $\begin{array}{c}c H_{c} / 4 \pi \lambda \\
\lambda: \text { penetration depth }\end{array}$ & $\begin{array}{l}\text { London theory; kinetic energy } \\
\text { density is equated to } \\
\text { condensation energy density }\end{array}$ & [2] p. 118 \\
\hline 3 & Type I; thin film or wire & $c H_{c}(T) / 3 \sqrt{6} \pi \lambda(T)$ & Ginsburg-Landau theory & [2] p. 117 \\
\hline 4 & Type I & $\begin{array}{c}e n_{s} \Delta / \hbar k_{F} \\
e: \text { electronic charge } \\
n_{s}: \text { density of superconducting electrons } \\
\Delta: \text { gap; } k_{F}: \text { Fermi wave vector }\end{array}$ & BCS theory & [3] p. 248 \\
\hline 5 & Type I; cylinder of radius $a$ & $\begin{array}{c}30 \Delta M / a \\
\Delta M: \text { width of magnetization loop at a given } \\
\text { field and temperature }\end{array}$ & Bean's critical state model & {$[4]$} \\
\hline 6 & Type I; slab of thickness $d$ & $\begin{array}{c}40 \Delta M / d \\
\alpha /\left(B+B_{0}\right)\end{array}$ & Bean's critical state model & [4] \\
\hline 7 & Type I; hollow cylinder & $\begin{array}{c}\alpha \text { and } B_{0} \text { (thermodynamic critical field) are } \\
\text { obtained from experiment }\end{array}$ & Kim et al. model & {$[5]$} \\
\hline
\end{tabular}

Fermi surfaces of a variety of high- $T_{c}$ SCs as reported in [e.g., 8,9] and, more recently, in [10-12]. In particular, in the latter of these references, the gaps observed in ironpnictide SCs as nodes or line nodes on the Fermi surface have evinced considerable interest. For a quantitative account of the $T_{c}$ and the multiple gaps of a prominent member of the iron-pnictide family, namely

$\mathrm{Ba}_{0.6} \mathrm{~K}_{0.4} \mathrm{Fe}_{2} \mathrm{As}_{2}$, in the framework of the generalizedBCS equations (GBCSEs) [13] — which will be further discussed below, we draw attention to [14].

The purpose of this note is to present an approach in which $P_{c}(T)$-defined as the momentum at which the binding energy of the CPs vanishes (this is equivalent to the vanishing of the gap [13]) — is calculated via the dynamics of CPs. As will be seen, we are then led via (1) to an equation for $j_{c}(T)$ directly in terms of the familiar BCS parameters, namely the dimensionless coupling constant $[\mathrm{N}(0) \mathrm{V}]$, the Debye temperature $\theta_{D}$ and, additionally, $E_{F}$ of the SC. The framework employed by us is that of the Bethe-Salpeter equation (BSE) for reasons to be spelled out shortly.

The paper is organized as follows. In the next section, we obtain equations for $P_{c}(T, H=0)$ and $P_{c}(T=0, H=0)$ for a simple SC. The solutions of these equations for $\mathrm{Sn}$ are obtained in Section 3 and compared with similar results obtained by a different method. Extension of our approach for non-elemental SCs is presented in Section 4. In Section 5 we make four brief comments. The final section sums up our conclusions.

\section{Equations for $P_{c}(T, H=0)$ and $P_{c}(T=0$, $\boldsymbol{H}=0$ ) for a Simple SC}

Our starting point is the $T=0, H=0 \mathrm{BSE}$ [15] for the bound states of particles $a, b$ bound via the interaction kernel $I_{a, b}^{c}$ in the ladder and instantaneous approximations:

$$
F_{a} F_{b} \psi\left(p_{\mu}\right)=(2 \pi i)^{-1} \int \mathrm{d}^{4} q_{\mu} \psi\left(p_{\mu}+q_{\mu}\right) I_{a, b}^{c}(\boldsymbol{q}) .
$$

Customization of this equation for CPs requires th at $a, b$ should be electrons. We then have

$$
\begin{aligned}
& F_{a}^{-1}=1 /\left[\gamma_{\mu}^{a} P_{\mu} / 2+\gamma_{\mu}^{a} p_{\mu}-m+i \varepsilon\right] \\
& F_{b}^{-1}=1 /\left[\gamma_{\mu}^{b} P_{\mu} / 2-\gamma_{\mu}^{b} p_{\mu}-m+i \varepsilon\right],
\end{aligned}
$$

where $\mathrm{m}$ is the electron mass, $\gamma_{\mu}^{a, b}$ are the Dirac matrices, $\pm p_{\mu}$ are 4-momenta of the two electrons in the centre of mass (c.m.) frame, and $P_{\mu}$ is the 4-momentum of the c.m. of the $\mathrm{CP}$ in the laboratory frame.

In our earlier work [13] based on (2), it sufficed to set

$$
P_{\mu}=(E, 0) ;\left(E=2 E_{F}+W\right)
$$

where $E$ is the total energy of a CP; it then turned out that $|W| \cong \Delta$. The BCS interaction kernel in (2) then was

$$
\begin{aligned}
& I_{a, b}^{c}(\boldsymbol{q}-\boldsymbol{p})=\frac{-V}{(2 \pi)^{3}}(V>0) \text { for } \\
& E_{F}-\omega_{D} \leq \frac{\boldsymbol{p}^{2}}{2 m}, \frac{\boldsymbol{q}^{2}}{2 m} \leq E_{F}+\omega_{D}
\end{aligned}
$$


[Note: We use natural units: mass, momentum, energy, etc. in $\mathrm{eV}, c=\hbar=1]$.

The role of the $4^{\text {th }}$ dimension in (2) is simply to provide the means to temperature-generalize the theory at the outset via the Matsubara recipe. Thus, following the steps that have been detailed in $[13,16]$ we obtain from (2) the 3-dimensional equation

$$
S(\boldsymbol{p})=(-2 \pi i)^{-1} \int \mathrm{d}^{3} q I_{a, b}^{c}{ }^{\prime}(\boldsymbol{q}-\boldsymbol{p}) S(\boldsymbol{q}) J(\boldsymbol{q}),
$$

where

$$
\begin{aligned}
& S(\boldsymbol{p})=\left[p_{4}+A(\boldsymbol{p})\right]\left[B(\boldsymbol{p})-p_{4}\right] \psi\left(p_{\mu}\right), \\
& A(\boldsymbol{p})=E / 2-\boldsymbol{p}^{2} / 2 m, \\
& B(\boldsymbol{p})=A(\boldsymbol{p}), \\
& I_{a b}^{c}(\boldsymbol{q}-\boldsymbol{p})=\gamma_{4}^{a} \gamma_{4}^{b} I_{a b}^{c}(\boldsymbol{q}-\boldsymbol{p}),
\end{aligned}
$$

and

$$
J(\boldsymbol{q})=\int \frac{\mathrm{d} q_{4}}{\left[q_{4}+A(\boldsymbol{q})\right]\left[B(\boldsymbol{q})-q_{4}\right]}
$$

If we simply carry out the integration in (8), we obtain the usual $T=0$ theory; subjecting it to the Matsubara recipe, however, we obtain an equation valid at any temperature - causing the theory to incorporate many-body effects. With the aid of the Matsubara recipe, (8) yields $[13,16]$ :

$$
J(\vec{q})=i \pi \frac{\left[\tanh \left\{\frac{\beta}{2} A(\vec{q})\right\}+\tanh \left\{\frac{\beta}{2} B(\vec{q})\right\}\right]}{D(\vec{q})},
$$

where

$$
D(\boldsymbol{q}) \equiv A(\boldsymbol{q})+B(\boldsymbol{q})=\left(E-\boldsymbol{q}^{2} / m\right),
$$

and $\beta=1 / k_{B} T, k_{B}$ being the Boltzmann constant.

Since critical velocity is defined as the velocity of CPs at which $W=0$, we now need to consider (2) for the case of moving CPs. Hence (4) is replaced by

$$
P_{\mu}=(E, \boldsymbol{p})
$$

where $\boldsymbol{p}$ is the 3 -momentum of the c.m. of a CP. It $\mathrm{i}$ $s$ pertinent at this stage to draw attention to the intera ction Hamiltonian corresponding to (2), which is actua lly apparent from the structure of the equation:

$$
H_{\mathrm{int}, B S E}=g \bar{\psi} \psi \varphi
$$

where $\psi$ is the electron field and $\varphi$ the phonon field; exchanges of the latter field between the electrons with coupling strength $g$ being responsible for pairing. Both for elemental and non-elemental SCs, one is now enabled to calculate not only the Tcs and $\Delta s$ - as has been shown $[17,18]$, but also $\operatorname{Pc}(T) s$ of the pairs as will be seen below. Because the BSE formalism accommodates CPS having non-zero c.m. momentum, it constitutes a larger framework than the original BCS formalism which restricts the Hamiltonian at the outset to comprise of terms corresponding to pairs having zero c.m. momentum.

Since energies of the electrons forming a $\mathrm{CP}$ now take on the values $(\boldsymbol{P} / 2 \pm \boldsymbol{p} \text { or } \pm \boldsymbol{q})^{2} / 2 m$, the BCS model interaction given in (5) gets replaced by

$$
\begin{aligned}
& I_{a b}^{c}(\boldsymbol{q}-\boldsymbol{p})=\frac{-V}{(2 \pi)^{3}}(V>0) \text { for } E_{F}-\hbar \omega_{D} \\
& \leq \frac{(\boldsymbol{P} / 2+\boldsymbol{p} \text { or } \boldsymbol{q})^{2}}{2 m}, \frac{(\boldsymbol{P} / 2-\boldsymbol{p} \text { or }-\boldsymbol{q})^{2}}{2 m} \leq E_{F}+\hbar \omega_{D} \\
& =0 \text { (otherwise). }
\end{aligned}
$$

Substituting (9)-(12) into (6), we obtain

$$
S(\boldsymbol{p})=\frac{V}{2} \int_{L}^{U} \frac{\mathrm{d}^{3} q}{(2 \pi)^{3}} \frac{T_{1}(\boldsymbol{q})+T_{2}(\boldsymbol{q})}{\operatorname{De}(\boldsymbol{q})} S(\boldsymbol{q})
$$

where

$$
\begin{aligned}
& T_{1}(\boldsymbol{q})=\tanh \left\{\beta\left[E / 2-(\boldsymbol{P} / 2+\boldsymbol{q})^{2}\right] / 2 m\right\}, \\
& T_{2}(\boldsymbol{q})=\tanh \left\{\beta\left[E / 2-(\boldsymbol{P} / 2-\boldsymbol{q})^{2}\right] / 2 m\right\}, \\
& \operatorname{De}(\boldsymbol{q})=\left[E-\frac{(\boldsymbol{P} / 2+\boldsymbol{q})^{2}}{2 m}-\frac{(\boldsymbol{P} / 2-\boldsymbol{q})^{2}}{2 m}\right]
\end{aligned}
$$

and it is seen that, as is well known for a constant kernel, the wave function for the pair is a constant; the limits (L, $\mathrm{U})$ will be dealt with shortly. Putting

$$
\frac{T_{1}(\boldsymbol{q})+T_{2}(\boldsymbol{q})}{D e(\boldsymbol{q})}=\varphi(\boldsymbol{q})
$$

in (13), multiplying the resulting equation with

$$
\int_{L}^{U} \mathrm{~d}^{3} p /(2 \pi)^{3}
$$

and simplifying, we obtain

$$
1=\frac{V}{2} \int_{L}^{U} \frac{\mathrm{d}^{3} p}{(2 \pi)^{3}} \cdot \frac{T_{1}(\boldsymbol{p})+T_{2}(\boldsymbol{p})}{\operatorname{De}(\boldsymbol{p})}
$$

with

$$
d^{3} p=p^{2} d p \sin \theta d \theta d \varphi, \xi=p^{2} / 2 m-E_{F},
$$

so that, since the integration range for $\xi \square E_{F}$,

$$
p \square\left(2 m E_{F}\right)^{1 / 2}, p^{2} d p \square m\left(2 m E_{F}\right)^{1 / 2} d \xi,
$$

we obtain from (14) the equation

$$
1=\frac{V}{8}\left[\frac{(2 m)^{3 / 2} E_{F}^{1 / 2}}{4 \pi^{2}}\right] \int_{-1}^{1} \mathrm{~d} x \int_{L}^{U} \mathrm{~d} \xi \frac{X}{Y}
$$

where 


$$
\begin{aligned}
& X=A_{1}+A_{2} \\
& A_{1}=\tanh \left[\frac{\beta}{2}\left(\xi+P \alpha x+\frac{P^{2}}{8 m}-\frac{W}{2}\right)\right] \\
& A_{2}=\tanh \left[\frac{\beta}{2}\left(\xi-P \alpha x+\frac{P^{2}}{8 m}-\frac{W}{2}\right)\right] \\
& Y=\xi+\frac{P^{2}}{8 m}-\frac{W}{2} \\
& \alpha=\left(\frac{E_{F}}{2 m}\right)^{1 / 2}, x=\cos (\boldsymbol{P}, \boldsymbol{p})
\end{aligned}
$$

and (13a) and the definition of $E$ in (4) have been used. In the natural units employed by us, both $\mathrm{m}$ and $\mathrm{E}_{\mathrm{F}}$ are in $\mathrm{eVs}$; the second pre-factor within the square brackets on the RHS of (16) is therefore recognized as the 3-dimensional density of states at the Fermi surface (with the dimensions of $\left(\mathrm{eV}^{-1} \cdot \mathrm{cm}^{-3}\right)$ in the units customarily employed in the BCS theory). Henceforth we denote this factor by $\mathrm{N}(0)$. Note that the term corresponding to $P$ p $x / 2 m$ in the expansion of $(\boldsymbol{P} / 2 \pm \boldsymbol{p})^{2} / 2 m$ has been written as $P \alpha x$ by using (15) and the definitions of $\alpha$ and $x$ that follow (16).

We now specify the limit $L$. It follows from (12) that

$$
E_{F}-\omega_{D} \leq \frac{p^{2}}{8 m}+\frac{p^{2}}{2 m}+P \alpha x, E_{F}-\omega_{D} \leq \frac{p^{2}}{8 m}+\frac{p^{2}}{2 m}-P \alpha x
$$

where (15) has been used. These relations may be written as

$$
\begin{aligned}
& -\omega_{D}-\frac{p^{2}}{8 m}-P \alpha x \leq \frac{p^{2}}{2 m}-E_{F} \equiv \xi \\
& -\omega_{D}-\frac{p^{2}}{8 m}+P \alpha x \leq \frac{p^{2}}{2 m}-E_{F} \equiv \xi .
\end{aligned}
$$

If we denote $\left(-\omega_{D}-\frac{p^{2}}{8 m}-P \alpha x\right)$ by point $A$, and $\left(-\omega_{D}-\frac{p^{2}}{8 m}+P \alpha x\right)$ by point $\mathrm{B}$ on the energy axis, then it follows that $\xi$ should always lie to the right of both $\mathrm{A}$, and $\mathrm{B}$. Thus $\mathrm{L}$ is fixed as

$$
L=-\omega_{D}-\frac{p^{2}}{8 m}+P \alpha x
$$

Similarly,

$$
U=\omega_{D}-\frac{p^{2}}{8 m}-P \alpha x .
$$

We now put $W=0$ in (16) in order to determine the critical momentum $P_{c}(T)$ at any temperature. Simultaneously, we neglect $P_{c}^{2} / 8 m$ everywhere-a posteriori justification to follow, excepting in the denominator of the integrand where it must be retained so as to avoid the singularity at $\xi=0$. It is then seen that it is an excellent approximation to write (16) as:

$$
1=\frac{[N(0) V]}{2} \int_{0}^{1} \mathrm{~d} x \int_{0}^{U^{\prime}} \mathrm{d} \xi \frac{\psi\left(x, \xi, P_{c}\right)}{\xi+P_{c}^{2} /(8 m)}
$$

where

$$
\begin{aligned}
\psi\left(x, \xi, P_{c}\right) & =\tanh \left[\frac{\beta}{2}\left(\xi+P_{c} \alpha x\right)\right]+\tanh \left[\frac{\beta}{2}\left(\xi-P_{c} \alpha x\right)\right] \\
& \equiv f_{1}\left(x, \xi, P_{c}\right)+f_{2}\left(x, \xi, P_{c}\right) \\
U^{\prime} & =\omega_{D}-P_{c} \alpha x .
\end{aligned}
$$

Equation (19) affords a consistency check of our procedure so far: Putting $P_{c}=0$ causes the $x$-integral to yield unity, and the two tanh-functions to add up, leading to the correct BCS equation for $T_{c}$. Note that when $T=0$ $(\beta=\infty), f_{1}\left(P_{c}, \xi, x\right)=1$, whereas the value of $f_{2}\left(P_{c}, \xi, x\right)$ depends on whether $\xi$ is less or greater than $P_{c} \alpha x$. Therefore, when $T=0$, we can write (19) as

$$
1-\frac{\lambda}{2}\left(I_{1}+I_{2}\right)=0
$$

where

$$
\begin{gathered}
I_{1}=\int_{0}^{1} \mathrm{~d} x \int_{0}^{E_{1} x-E_{2}} \frac{d \xi}{\xi+E_{3}}, \\
I_{2}=\int_{0}^{1} \mathrm{~d} x\left[\int_{0}^{E_{2} x} \frac{-\mathrm{d} \xi}{\xi+E_{3}}+\int_{E_{2} x}^{E_{1}-E_{2} x} \frac{\mathrm{d} \xi}{\xi+E_{3}}\right], \\
\lambda=[N(0) V], E_{1}=\omega_{D}, E_{2}=P_{0} \alpha, \\
\mathrm{P}_{0}=P_{c}(T=0), \mathrm{E}_{3}=P_{0}^{2} / 8 m .
\end{gathered}
$$

Carrying out the elementary integrations in (22) and (23), (21) yields

$$
\begin{array}{r}
1-\lambda\left[\frac{E_{1}+E_{3}}{E_{2}} \ln \left(\frac{E_{1}+E_{3}}{E_{2}}\right)+\frac{E_{3}}{E_{2}} \ln \left(\frac{E_{3}}{E_{2}+E_{3}}\right)+\right. \\
\left.+\ln \left(\frac{E_{1}-E_{2}+E_{3}}{E_{2}+E_{3}}\right)\right]=0 .
\end{array}
$$

Since, as will be seen below, $E_{3} \square E_{1}, E_{2}$, we may write it more compactly as

$$
1-\lambda\left[y \ln \left(\frac{y}{y-1}\right)+\ln (y-1)\right]=0,
$$

where the dimensionless parameter

$$
y=E_{1} / E_{2}=\omega_{D} / P_{0} \alpha .
$$

\section{Solutions of (25) and (19) for Sn and Comparison of Results for $\boldsymbol{j}_{c}$ via (1) with Those Obtained via an Alternate, Indirect Approach}

We deal with $\mathrm{Sn}$ because superconducting properties based on its $j_{\mathrm{c}}$ have been discussed in standard texts such 
as [3; p. 248] and [19; p. 138]. The equation invoked for $j_{c}$ at $T=0$ in these texts is:

$$
j_{c}=\frac{e n_{s} \Delta}{p_{F}}
$$

where $n_{s}$ is the number of electrons (not pairs) and $p_{F}$ the Fermi momentum. Indeed this equation is equivalent to using (1) since $\Delta / p_{F}$ has the dimensions of velocity. With $\Delta=1.80 k_{B} T_{c}\left(T_{c}=3.72 \mathrm{~K}\right), m^{*}=1.26 x$ free electron mass $\left[20\right.$, p. 254] and $v_{F}$ taken at the Fermi edge to be $6.97 \times 10^{7} \mathrm{~cm} / \mathrm{sec}$ [3, p. 248], we have

$$
\begin{aligned}
v_{c}=\Delta / p_{F} & =\Delta / m * v_{F}=1.46 \times 10^{4} \mathrm{~cm} \cdot \mathrm{sec}^{-1} ; \\
\mathrm{E}_{F} & =(1 / 2) m * v_{F}^{2}=1.74 \mathrm{eV},
\end{aligned}
$$

Using (27) and the experimental value of $j_{c}$ for Sn $(\sim 2$ $\times 10^{7}$ Ampere $\left.\mathrm{cm}^{-2}\right),(26)$ is invoked to calculate $n_{s}$ since it is the most uncertain quantity in the equation. It is thus found that

$$
n_{s}=8.50 \times 10^{21} \mathrm{~cm}^{-3},
$$

which, it has been remarked [19], is appreciably less than one electron per atom, but not unreasonable in view of the complicated band structure of tin, which has been discussed in [7, p. 294].

In our approach, we first need the value of $\lambda$ to solve (25). Substituting the experimental value of $T_{c}$ quoted above and $\theta_{D}=195 \mathrm{~K}$ in the BCS equation for $T_{c}$ : $\lambda=-1 / \ln \left(T_{c} / 1.14 \theta_{D}\right)$, we obtain $\lambda=0.2445$, whence (25) yields

$$
y=\frac{\omega_{D}}{P_{0} \alpha}=\frac{k_{B} \theta_{D}}{P_{0}} \sqrt{\frac{2 m^{*}}{E_{F}}}=22.48,
$$

where the definition of $\alpha$ given after (16) has been used. Using (29) and (1), we have

$$
\begin{aligned}
& v_{0}=\frac{P_{0}}{2 m^{*}}=A \sqrt{\frac{B}{E_{F}}} \\
& \mathrm{~A}=\left(\frac{k_{B} \theta_{D}}{2 m^{*} c y}\right)=17.402 \mathrm{~cm} \mathrm{sec}^{-1}, \\
& \mathrm{~B}=2 \times 1.26 \times 0.5110 \times 10^{6} \mathrm{eV}
\end{aligned}
$$

and

$$
j_{0}=n_{s} e^{*} A \sqrt{\frac{B}{E_{F}}},
$$

where $2 m^{*}$ is the effective mass of a CP and $m^{*}$ has been taken to be 1.26 times the free electron mass as before, $e^{*}$ is twice the electronic charge and the value of $E_{F}$ is in $\mathrm{eV}$.

Since we have already determined $A$ via dynamics of the problem, $e^{*}$ and $\mathrm{B}$ are known constants and $j_{0}$ is known from experiment, (31) involves two unknowns: $n_{s}$ and $E_{F}$; knowledge of either of them enables one to calculate the other. Guided by text book wisdom, if we use the values of $j_{0}\left(2 \times 10^{7} \mathrm{Amp} \mathrm{cm}{ }^{-2}\right)$ and $E_{F}$ (given in (27)), we obtain from (30) and (31) the following results

$$
\begin{gathered}
\alpha=1.162 \times 10^{-3}, \mathrm{P}_{0}=0.643 \mathrm{eV} \\
\mathrm{E}_{1}=1.68 \times 10^{-3} \mathrm{eV}, \mathrm{E}_{2}=7.476 \times 10^{-4} \mathrm{eV}, \\
\mathrm{E}_{3}=8.029 \times 10^{-8} \mathrm{eV} \\
v_{0}=1.50 \times 10^{4} \mathrm{~cm} \mathrm{sec}^{-1} \\
n_{s}(\mathrm{CPs})=4.17 \times 10^{21} \mathrm{~cm}^{-3} .
\end{gathered}
$$

The values of $E_{1}, E_{2}$ and $E_{3}$ in (32) justify the approximation made in reducing (24) to (25). The result in (33) is almost identical with the value obtained via (26) and quoted in (27), while the result in (34) translates into $8.34 \times 10^{21} \mathrm{~cm}^{-3}$ for the number of super electrons which, again, is in excellent agreement with the value quoted in (28). It is thus established that the approach followed in this paper provides an ab initio theoretical justifi- cation for the text book equation (26) valid at $T=0$; additionally: 1) it relates $\mathrm{j}_{\mathrm{c}}$ with the relevant parameters of the problem at $T \neq 0$ via (19) and 2) it can easily be extended to bring non-elemental high- $T_{c}$ SCs under its purview as will be discussed in the next section.

With $P_{0}$ known, it is convenient to solve (19) in terms of the reduced (or normalized) variables defined as $t=T / T_{c}$ and $p(t)=P_{c}(t) / P_{0}$. Figure 1 gives the results of this exercise for $0 \leq t \leq 1$. We have also studied the variation of $p$ with $\mathrm{t}$ for five other elements: $\mathrm{Pb}, \mathrm{Hg}$, $\mathrm{In}, \mathrm{Tl}$, and $\mathrm{Nb}$ - by taking for their $\mathrm{E}_{\mathrm{F}} \mathrm{S}$ the values given by the free electron model [20, p. 248], and found it to be similar to that of Sn.

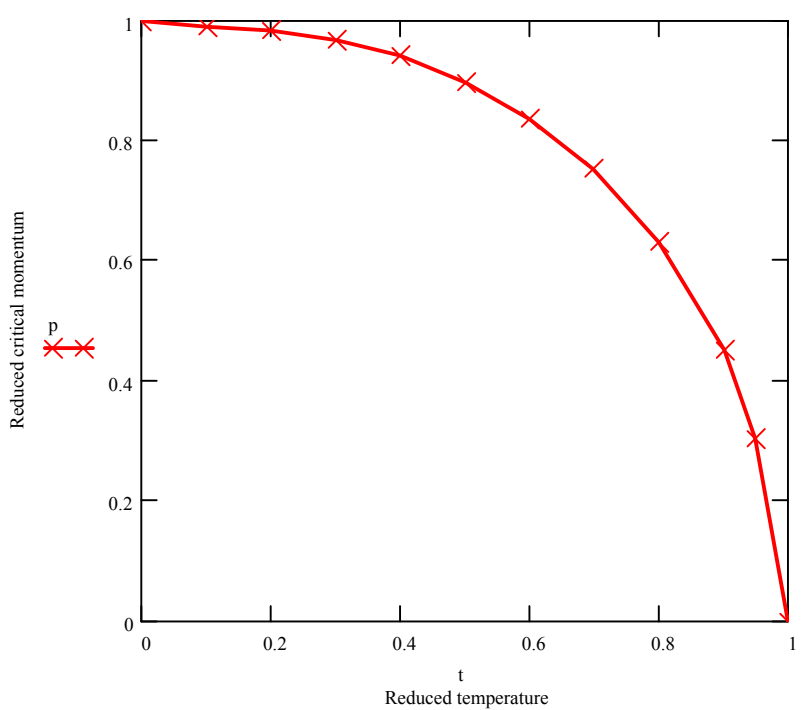

Figure 1. Variation of reduced critical momentum with reduced temperature for Sn obtained via (19) with the input of $\lambda=0.2445, \theta_{D}=195 \mathrm{~K}$ and $E_{F}=1.74 \mathrm{eV}$. 


\section{Equations for $\operatorname{Pc}(T, H=0)$ and $P c(T=0$, $H=0$ ) for a Non-Elemental SC}

The $T_{c} \mathrm{~s}$ and the multiple gaps of several non-elemental high- $T_{c}$ SCs (other than iron-pnictide SCs) have been dealt with in $[17,18]$ via GBCSEs. We recall from $[13,16]$ that these equations constitute a generalization of the BCS equations because: 1) they incorporate the mechanism of multi-phonon exchanges for the formation of Cooper pairs besides the usual one-phonon exchange mechanism; and 2) they invoke more than one Debye temperature-which is another way to specify the massdependent Debye frequency of an ion species - to characterize the $\mathrm{SC}$.

In order to calculate $P_{c}$ in the scenario in which CPs are bound via say, two-phonon exchange mechanism in a CS $A_{x} B_{1-x}$, we need to generalize (19) and (24). This is accomplished by replacing the propagator in (12) by a superpropagator [13]:

$$
\begin{aligned}
& I_{a b}^{c}(\boldsymbol{q}-\boldsymbol{p})=-\frac{\left(V_{1}^{c}+V_{2}^{c}\right)}{(2 \pi)^{3}}, \\
& \text { range of } \mathrm{V}_{1,2}^{c}: E_{F}-\left(\omega_{D}\right)_{1,2}^{c} \\
& \leq \frac{\left(\frac{\boldsymbol{P}}{2}+\boldsymbol{p} \text { or } \boldsymbol{q}\right)^{2}}{2 m}, \frac{\left(\frac{\boldsymbol{P}}{2}-\boldsymbol{p} \text { or }-\boldsymbol{q}\right)^{2}}{2 m} \\
& \leq E_{F}+\left(\omega_{D}\right)_{1,2}^{c} \\
& =0,(\text { otherwise })
\end{aligned}
$$

where $V_{1,2}^{c}>0$ are the BCS model interactions for the species of phonons belonging to $\mathrm{A}, \mathrm{B}$ in the combined state of the constituents $\mathrm{A}$ and $\mathrm{B}$, to be distinguished from $V_{1,2}^{c}$, which are the free state interactions of $A, B ;\left(\omega_{D}\right)_{1,2}^{c}$ are to be similarly distinguished from $\left(\omega_{D}\right)_{1,2}^{c}$. Following now the sequence of steps between (8) and (24), we obtain the generalized version of (19) as:

$$
1=\int_{0}^{1} \mathrm{~d} x\left[J_{1}(x)+J_{2}(x)\right],
$$

where

$$
\begin{aligned}
& J_{1}(x)=\frac{\lambda_{1}^{c}}{2} \int_{0}^{U_{1}} \mathrm{~d} \xi \frac{\psi\left(x, \xi, P_{c}\right)}{\xi+p_{c}^{2} / 8 m} \\
& J_{2}(x)=\frac{\lambda_{2}^{c}}{2} \int_{0}^{U_{2}} \mathrm{~d} \xi \frac{\psi\left(x, \xi, P_{c}\right)}{\xi+p_{c}^{2} / 8 m} \\
& \psi\left(x, \xi, P_{c}\right)=\tanh \left[\frac{\beta}{2}\left(\xi+P_{c} \alpha x\right)\right]+\tanh \left[\frac{\beta}{2}\left(\xi-P_{c} \alpha x\right)\right] \\
& \lambda_{1,2}^{c}=[N(0) V]_{1,2}^{c}, \mathrm{U}_{1,2}=\left(\omega_{D}\right)_{1,2}^{c}-P_{c} \alpha x .
\end{aligned}
$$

Equation (24) now goes over to

$$
\lambda_{1}^{c} \phi_{1}\left(\left(\omega_{\mathrm{D}}\right)_{1}^{c}, \alpha, p_{0}\right)-\lambda_{2}^{c} \phi_{2}\left(\left(\omega_{\mathrm{D}}\right)_{2}^{c}, \alpha, p_{0}\right)=0,
$$

where

$$
\begin{gathered}
\phi_{1}=\left[\frac{E_{1}}{E_{2}} \ln \left(\frac{E_{1}}{E_{1}-E_{2}}\right)+\frac{P_{0}}{8 m \alpha} \ln \left(\frac{P_{0}}{8 m \alpha}\right)+\right. \\
\left.+\ln \left(\frac{E_{1}-E_{2}}{E_{2}}\right)\right] \\
E_{1}=\left(\omega_{D}\right)_{1}^{c}, E_{2}=P_{0} \alpha ;
\end{gathered}
$$

$\phi_{2}$ is obtained from $\phi_{1}$ by putting $E_{1}=\left(\omega_{D}\right)_{2}^{c}$.

The solution of (39) for $\mathrm{MgB}_{2}$, for example, requires the inputs of $\lambda_{1,2}^{c}$ and the two Debye temperatures: $\left(k_{B} \theta_{D}\right)_{1,2}^{c}=\left(\omega_{D}\right)_{1,2}^{c}$; in addition, we require $E_{F}$ of the CS. Such solutions will be addressed else where n puts of $\lambda_{1,2}^{c}$ and the two Debye temperatures: $\left(k_{B} \theta_{D}\right)_{1,2}^{c}=\left(\omega_{D}\right)_{1,2}^{c}$; in addition, we require $E_{F}$ of the CS. Such solutions will be addressed elsewhere.

\section{Discussion}

We have dealt above with equations that were obtained via positive energy projection operators (PEPOs). This suffices for the problem addressed because $P_{c}$ corresponds to the situation when $W=0$; in this limit, it has been shown in [21] that the equation obtained via the negative energy projection operators is identical with the one obtained via the PEPOs; also that: 1) CPs formed via electron-electron and hole-hole scatterings make equal contributions to the BS amplitude; and 2) the amplitudes for the formation of CPs corresponding to the mixed energy projection operators are zero.

Note that if we concern ourselves with the ratios of $j_{c} \mathrm{~s}$ at different temperatures, which seems to be a realistic application of our equations, then the choice of the effective mass of the electron in (1) becomes immaterial.

Even a cursory survey of the literature shows that $j_{c}$ of an elemental/non-elemental SC can vary between wide limits - depending upon the shape, size and alloying materials of the sample. The study presented here suggests that this variation comes about because each sample has its own set of intrinsic parameters: $T_{c}, \theta_{D}$, and $E_{F}$. Substituting these into the equation for $j_{c}$ (which is known from experiment) leads to a relation involving $n_{s}$ and $E_{F}$. Knowledge of either of them then determines the other.

We finally note that the equations for $j_{c}(T)$ presented in this paper can be generalized to include an external magnetic field via the Landau quantization scheme - as has been done to obtain dynamics-based equations for critical magnetic fields for both elemental and non-elemental 
SCs in [22].

\section{Conclusions}

Equation (19) for an elemental SC and (36) for a nonelemental SC are the new results of this paper: they enable one to calculate the critical momentum $P_{c}$ of the SC at any $T$ in zero-external magnetic field directly in terms of the familiar parameters $[\mathrm{N}(0) \mathrm{V}], \theta_{D}$ and $E_{F}$ that characterize it. Substitution of these values of $P_{c}$ into (1) then constitutes a direct approach for the calculation of the critical current densities.

$T=0$ limits of both (19) and (36) were obtainedleading to (24) and (40), respectively. While it was further shown that (24) can justifiably be reduced to (25), we note that caution needs to be exercised should one seek to carry out a similar reduction of (40).

A necessary input for the calculation of $P_{c}$ (and hence $j_{c}$ ) of an SC is its $E_{F}$, which is a parameter that is seldom available for the high- $T_{c}$ SCs. It therefore seems to us that an immediate and realistic application of the approach presented here is to calculate the $E_{F}$ of such SCs via the input of their $\mathrm{j}_{\mathrm{c}} \mathrm{s}$ which are readily available in the literature. The importance of $E_{F}$ of the high- $T_{c}$ SCs is borne out by the studies reported in [6] and [8-12].

\section{Acknowledgements}

The author acknowledges correspondence with Professor M. de Llano and Professor D. C. Mattis having a bearing on this submission. He is grateful to Professor A. N. Mitra for continued encouragement.

\section{REFERENCES}

[1] M. Randeria, "Crossover from BCS Theory to Bose-Einstein Condensation," In: A. Griffin, D. W. Snoke and S. Stringari, Eds., Bose-Einstein Condensation, Cambridge University Press, Cambridge, 1995, p. 355. doi:10.1017/CBO9780511524240.017

[2] M Tinkham, "Introduction to Superconductivity," McGraw Hill, New York, 1975.

[3] H. Ibach and H. Lüth, "Solid State Physics," Springer, Berlin, 1996. doi:10.1007/978-3-642-88199-2

[4] C. P. Bean, "Magnetization of High-Field Superconductors," Reviews of Modern Physics, Vol. 36, No. 1, 1964, pp. 31-39. doi:10.1103/RevModPhys.36.31

[5] Y. B. Kim, C. F. Hempstead and A. R. Strand, "Magnetization and Critical Supercurrents," Physical Review, Vol. 129, No. 2, 1963, pp. 528-535. doi:10.1103/PhysRev.129.528

[6] A. S. Alexandrov, "Nonadiabatiic Superconductivity in MgB2 and Cuprates," 2001. http://arxiv.org/abs/cond-mat/0104413
[7] A. P. Cracknell and K. C. Wong, "The Fermi Surface," Clarendon Press, Oxford, 1973.

[8] J. C. Campuzano, G. Jennings, M. Faiz, L. Beaulaigue, B. W. Veal, J. Z. Liu, A. P. Paulikas, K. Vandervoort, H. Claus, R. S. List, A. J. Arko and R. J. Bartlett, "Fermi Surfaces of $\mathrm{YBa}_{2} \mathrm{Cu}_{3} \mathrm{O}_{6.9}$ as seen by Angle-Resolved Photoemission," Physical Review Letters, Vol. 64, No. 19, 1990, pp. 2308-2311. doi:10.1103/PhysRevLett.64.2308

[9] C. G. Olsson, R. Liu, D. W. Lynch, R. S. List, A. J. Arko, B. W. Veal, P. Z. Jiang and A. P. Paulika, "High-Resolution Angle-Resolved Photoemission Study of the Fermi Surface and the Normal-State Electronic Structure of $\mathrm{Bi}_{2} \mathrm{Sr}_{2} \mathrm{CaCu}_{2} \mathrm{O}_{8}$," Physical Review B, Vol. 42, No. 1, 1990, pp. 381-386. doi:10.1103/PhysRevB.42.381

[10] D.-H. Lee, "Iron-Based Superconductors: Nodal Rings," Nature Physics, Vol. 8, 2012, pp. 364-365. doi:10.1038/nphys2301

[11] Y. Zhang, Z. R. Ye, Q. Q. Ge, F. Chen, J. Jiang, M. Xu, B. P. Xie and D. L. Feng, "Nodal Superconducting-Gap Structure in Ferropnictide Superconductor $\mathrm{BaFe}_{2}\left(\mathrm{As}_{0.7} \mathrm{P}_{0.3}\right)_{2}$," Nature Physics, Vol. 8, 2012, pp. 371-375. doi:10.1038/nphys 2248

[12] M. P. Allan, A. W. Rost, A. P. Mackenzie, Y. Xie, J. C. Davis, K. Kihou, C. H. Lee, A. Iyo, H. Eisaki and T.-M. Chuang, "Anisotropic Energy Gaps of Iron-Based Superconductivity from Intraband Quasiparticle Interference in LiFeAs," Science, Vol. 336, No. 6081, 2012, pp. 563-567. doi:10.1126/science. 1218726

[13] G. P. Malik, "On the Equivalence of the Binding Energy of a Cooper Pair and the BCS Energy Gap: A Framework for Dealing with Composite Superconductors," International Journal of Modern Physics B, Vol. 24, No. 9, 2010, pp. 1159-1172. doi:10.1142/S0217979210055408

[14] G. P. Malik, I. Chávez and M. de Llano, "Generalized BCS Equations and Iron-Pnictide Superconductors," Journal of Modern Physics, Vol. 4, 2013, pp. 474-480.

[15] E. E. Salpeter, "Mass Corrections to the Fine Structure of Hydrogen-Like Atoms," Physical Review, Vol. 87, No. 2, 1952, p. 328. doi:10.1103/PhysRev.87.328

[16] G. P. Malik and U. Malik, "High-T $\mathrm{T}_{\mathrm{c}}$ Superconductivity via Superpropagators," Physica B, Vol. 336, No. 3-4, 2003 , pp. 349-352. doi:10.1016/S0921-4526(03)00302-8

[17] G. P. Malik, "Generalized BCS Equations: Applications," International Journal of Modern Physics B, Vol. 24, No. 19, 2010, pp. 3701-3712. doi: $10.1142 / \mathrm{S} 0217979210055858$

[18] G. P. Malik and U. Malik, "A Study of the Thallium- and Bismuth-Based High-Temperature Superconductors in the Framework of the Generalized BCS Equations," Journal of Superconductivity and Novel Magnetism, Vol. 24, No. 1-2, 2011, pp. 255-260. doi:10.1007/s10948-010-1009-0

[19] A. C. Rose-Innes and E. H. Rhoderic, "Introduction to Superconductivity," Pergamon, Oxford, 1978.

[20] C. Kittel, "Introduction to Solid State Physics," Wiley Eastern, New Delhi, 1974.

[21] G. P. Malik, "High- $T_{c}$ Superconductivity via Superpro- 
pagators Revisited," Physica C, Vol. 468, No. 13, 2008, pp. 949-954. doi:10.1016/j.physc.2008.03.002

[22] G. P. Malik, "On Landau Quantization of Cooper Pairs in a Heat Bath," Physica B, Vol. 405, 2010, pp. 3475-3481. doi:10.1016/j.physb.2010.05.026 